\title{
ROSENZWEIG, SCHELLING ET L'HISTOIRE: QUELQUES APERÇUS
}

ROSENZWEIG, SCHELLING E A HISTORIA: ALGUMAS PERSPECTIVAS

\section{Gérard Bensussan* gerard.bensussan@sfr.fr}

RESUME: Le rapport Rosenzweig / Schelling est le plus souvent, et à bon droit, étudié à partir de I'influence des Âges du monde sur l'écriture de l'Etoile de la rédemption. Le présent texte suggère une autre voie, non exclusive de la première, constituée autour de la dette rosenzweigienne contractée à l'endroit de la pensée schellingienne de l'historicité. Cette étude retrace les contenus et les attendus de cette dette sous quatre registres: $\mathbf{1}$. L'histoire, chez Schelling, est soumise à sa disposition comme articulation de temporalités sédimentées et coexistantes; 2 . Une véritable pensée de l'histoire peut être ainsi déterminée, très différente d'une " philosophie de l'histoire " : elle accueille l'histoire comme ce par quoi se fait connaître à l'expérience un imprépensable ; 3 . Cette histoire "expériencielle " se surprend sans cesse en effectuant l'événement de ce qui ne cesse d'advenir dans sa Wirklichkeit ; 4. L'acte fondatif et inaugural de l'histoire est un mouvement que les hommes ne peuvent reconnaître comme quelque chose qu'ils auraient accompli. L'histoire avance, mais sans voir. Elle s'avance sans se voir selon un "progrès anti-historique ". Le parcours de Rosenzweig refait à sa façon ce chemin. II va d'une conception néo-hégélienne de l'histoire universelle à l'idée d'une histoire discontinue, et même d'une méta- ou d'une extrahistoricité, c'està-dire d'une d'extériorité à l'histoire sans laquelle celle-ci s'aplatirait dans sa pure immanence et ne se laisserait pas penser dans sa concrétude opaque et chaotique.

MoTS-CLES: Absolu, christianisme, dialectique, effectivité, fin de l'histoire, fondement, Hegel, histoire, historialité, historicité, judaïsme, ontologie de I'histoire, philosophie de l'histoire, révélation, Rosenzweig, Schelling, temporalité(s).

RESUMO: A relação Rosenzweig/Schelling é mais estudada e legitimamente a partir da influência das Idades do Mundo na escrita de $A$ Estrela da Redenção. O presente texto sugere uma outra via, não exclusiva da primeira, constituída em torno da dívida rosenzweiguiana contraída no contacto com o pensamento schellinguiano da historicidade. Este estudo segue os conteúdos e as expectativas desta dívida sob quatro registos : 1. A História, em Schelling, encontra-se submetida à sua disposição como articulação de temporalidades sedimentadas e coexistentes; 2 . Um verdadeiro pensamento da história pode ser assim determinado, muito diferente de uma "filosofia da história»: ela acolhe a história como aquilo pelo qual se dá a conhecer na experiência como um não pré-pensável; 3. Esta história «experiencial» surpreende sem cessar, efectuando o acontecimento do que não pára de advir, na sua Wirklichkeit; 4. O acto fundador e inaugural da história é um movimento que os homens não podem reconhecer senão como qualquer coisa se não fosse cumprido. A história avança, mas sem ver. Ela avança sem se ver de acordo com um "progresso anti-histórico». 0 percurso de Rosenzweig refaz, à sua maneira, este caminho. Ele vai de uma concepção neo-hegeliana da história universal à ideia de uma história descontínua e, mesmo, de uma meta ou de uma extrahistoricidade, ou seja, de uma exterioridade à história sem a qual esta se achataria na sua pura imanência e não se deixaria pensar na sua concretude opaca e caótica.

PALAVRAS-CHAVE: Absoluto, cristianismo, dialéctica, efectividade, fim da história, fundamento, Hegel, história, historialidade, historicidade, judaísmo, ontologia da história, filosofia da história, revelação, Rosenzweig, Schelling, temporalidade(s).

\footnotetext{
* Professor de Filosofia na Université Marc Bloch (Strasbourg) e Investigador do CNRS.
} 
Le rapport de Rosenzweig à Schelling a déjà fait l'objet d'études et d'enquêtes minutieuses, suscitées d'ailleurs par ce qu'en a dit l'auteur de l'Etoile le tout premier à propos de sa découverte des Âges du Monde dans l'édition Reclam de 1913, à partir aussi des commentaires toujours élogieux qui émaillent la correspondance, sans rien dire de son travail académique d'édition, de publication et d'attribution à Schelling d'un texte de la main de Hegel, Le plus ancien programme systématique de l'idéalisme allemand. II y a donc, constitué et consistant, un rapport Schelling / Rosenzweig, avec à son avers en quelque sorte le rapport à Hegel.

Son examen se concentre le plus souvent, et à bon droit, sur la reprise, dès la Urzelle, puis dans l'Etoile, de la structure disposée dans les esquisses des Âges $d u$ Monde, reprise assumée jusqu'à son terme puisque Rosenzweig affirme avoir mené le projet schellingien, abandonné au seul chantier du passé, jusqu'à une manière d'accomplissement à travers l'investigation des deux autres âges. Les Âges entendaient raconter, au sens strict d'une philosophie narrative, l'histoire de Dieu, d'un Dieu en devenir, comme dit Schelling, soit d'un Dieu qui passe à l'existence historique, d'un Absolu qui transit l'histoire, et qui, ce faisant, se révèle dans le temps, dans les trois temps absolus que sont le passé, le présent et le futur éternels, c'est-à-dire non pas successifs, successivement distribués dans I'homogénéité d'une histoire, mais stratifiés en profondeur, co-existant dans la conscience. Les "grands temps" du monde figurent alors dans leur organicité le temps vécu des hommes. Ce schéma se retrouve dans l'Etoile, laquelle, à sa façon, raconte également une histoire de l'être à travers ses trois "âges», le passé immémorial, l'existence dans la révélation et l'éternité anticipée. Ces temps absolus (Création / Révélation / Rédemption) renvoient, comme chez Schelling, à un temps ou des temps expériencés, à des temps existentiels, le passé comme expérience de la permanence, le présent comme expérience de son incessant renouvellement, le futur comme expérience d'une anticipation (éventuellement impossible ou incessamment différée comme dans "l'expérience » messianique). Ce qui est donc commun aux Âges du Monde et à l'Etoile de la Rédemptionquelles que puissent être les modalités différentes de la réalisation, avec le recentrage sur la révélation opéré par Rosenzweig, comme il l'affirme lui-même (ROSENZWEIG, Foi et Savoir 
2001, 103) ${ }^{1}$ - c'est, au fond, ce schéma d'une historiosophie qui supporterait la pensée d'une temporalité existentielle, extatique, organique et dynamique. La question de l'histoire comme histoire de l'Absolu constitue donc l'accès le plus éminent à l'examen du rapport Schelling / Rosenzweig.

Je voudrais tenter d'esquisser ou de suggérer une autre voie, par où apparaîtrait également, toujours autour de la question de l'histoire et de l'historicité, la dette rosenzweigienne, mais sur un autre versant et autrement constituée. Le parcours de Rosenzweig, on le voit dans la correspondance, avec Rosenstock surtout, le conduit d'une conception hégélienne ou néo-hégélienne de l'histoire universelle, qu'on trouve encore dans la thèse et qui s'écrit sous influence, celle de Meinecke en particulier, vers l'idée d'une histoire discontinue, scandée par des occurrences uniques et signifiantes qui en dessineraient secrètement la ligne invisible, sous l'effet peut-être aussi de la prise en considération de la chronosophie rosenstockienne. Ce cheminement le mènera à la grande thèse, affirmée à partir de l'Etoile et dans les textes postérieurs, d'une métahistoricité qui serait proprement une extra-historicité, c'est-à-dire d'une dimension d'extériorité à l'histoire sans laquelle celle-ci s'aplatirait dans sa pure et lisse immanence et, du coup, ne se laisserait pas penser dans sa concrétude opaque et chaotique.

Je parlais de dette. Je crois en effet que la dernière philosophie de Schelling fournit les thèmes, les linéaments peut-être, de la thèse rosenzweigienne d'un autre de toute histoire manifesté historiquement par des configurations particulières et condensées d'un rapport histoire/éternité -par où s'esquisse donc la quête d'une articulation des grands temps et du temps vécu. Pour essayer de le montrer, je dois revenir aussi précisément que possible à la pensée et au texte de Schelling lui-même, en supposant plus ou moins connue la thèse rosenzweigienne, que je rappellerai cependant et sur laquelle je reviendrai en conclusion pour indiquer en quoi, dans leurs dissemblances mêmes, les deux positions entrent en un rapport « stellaire».

Philosophie historique et philosophie de l'histoire : l'usage des termes

Je voudrais commencer par faire rapidement le point sur l'usage ou les usages des termes histoire, historique, historicité, dans la ou les philosophie(s) de Schelling. Je ne le

\footnotetext{
${ }^{1}$ On peut considérer que ce recentrage est effectué par Schelling lui-même, à sa façon, dans la dernière philosophie.
} 
ferai pas en en retraçant les avatars et le cheminement sinueux depuis les premiers textes, ou tout au moins depuis le Système de l'idéalisme transcendantal, jusqu'à la dernière philosophie, ce qu'autoriserait pleinement la "tendance à l'historique » que Schelling croit lui-même rétrospectivement distinguer dans toute sa production philosophique et dans ce qui aura été sa façon la plus propre de philosopher ${ }^{2}$. Je me situerai plutôt et d'emblée du point de vue de la dernière philosophie, c'est-à-dire du point de vue d'une philosophie dite par son auteur « historique ».

Il faut rappeler en effet que la Spätphilosophie s'efforce de faire coïncider sa " positivité » avec ce caractère historique, tel que Schelling l'avait déterminé dans son enseignement des années munichoises. La philosophie positive est historique -et le contraste se trace par rapport à la philosophie négative, dite, elle, "logique » ou " purement rationnelle ", et en tant que telle " anhistorique ". Mais alors qu'est-ce qui est historique dans la philosophie positive ? II peut sembler y avoir là une difficulté ou au moins une trop hâtive identification. Le cours de Munich de 1830 (SCHELLING, Introduction à la philosophie 1996) cerne cette difficulté comme telle. II s'agirait d'“ écarter les éventuels malentendus ॥, dit Schelling, sans doute en réponse à une question ou une objection formulée par un auditeur entre les leçons IV et $V$, d'une séance à la suivante donc. II suggère qu'il lui faudra peut-être finalement laisser tomber l'expression de philosophie historique et la « remplacer » par celle de philosophie positive. Ce qu'il fera tendanciellement si on compare la fréquence des occurrences. Mais notons-le, l'expression de philosophie historique ne disparaît jamais. Schelling n'y renonce en aucune façon et évoque « celle que nous tenons toujours à nommer encore philosophie historique » (SCHELLING, Introduction à la philosophie 1996, 32).

Je résume l'argument de l'Introduction de 1830 qu'une longue note de I'Introduction de Berlin de 1841 synthétisera de façon très ramassée. Par deux fois en tout cas, l’argument est négatif.

Schelling insiste en effet sur ce que n'est pas et que ne saurait signifier historique dans l'expression "philosophie historique » en tant que celle-ci, c'est-à-dire la philosophie positive, philosophie de la mythologie et philosophie de la révélation, se contre-distingue de

\footnotetext{
${ }^{2}$ SCHELLING, Contribution à l'histoire de la philosophie moderne, trad. J.F. Marquet, PUF, 1983, p. 110; SW, X, p. $94:$ : „so verriet sich schon durch meine ersten Schritte in der Philosophie die Tendenz zum Geschichtlichen“ (souligné par moi).
} 
tout savoir des seules nécessités internes à la sphère de la pensée. Pour cette raison même, elle ne saurait être une histoire de la philosophie qui suivrait la succession chronologique contingente des systèmes ${ }^{3}$. Qui se contente de penser sur la pensée cesse par là-même de penser (SCHELLING, Introduction à la philosophie 1996, 38). II ne faut pas la prendre, dit ensuite Schelling, pour une " philosophie de l'histoire ", ce qui, ajoute-t-il lapidairement, va encore davantage de soi. Elle n'est pas non plus un savoir qui procèderait de la seule révélation et renoncerait aux instruments de la raison. Ni histoire de la philosophie, donc, ni « philosophie de l'histoire », ni « philosophie subjective », comme il dit aussi.

Ce qui fait que la philosophie positive est historique, ce n'est donc pas le mode de traitement, à vrai dire, mais son objet même, ce n'est pas le "mode du savoir ", mais son "contenu», comme le précise la note berlinoise de 1841. En d'autres termes: une philosophie qui chercherait à montrer que l'être ne peut être trouvé qu'à condition d'admettre " quelque chose de positif, la volonté, la liberté, le fait », et de "se diriger vers quelque chose d'extérieur", pareille philosophie serait en vérité celle qui suit "la voie historique " (Ibidem, 37, 38, 30). Il est évident que Schelling prend ici en vue rétrospective le basculement qui s'est joué dans son propre parcours philosophique, soit dans sa philosophie médiane, le passage d'un primat de l'ontologique à une subordination à l'historique, de l'anhistoricité de l'essence à l'explication par l'acte. Cette détermination limitative et ses attendus descriptifs demeurent assez flous dans l'Introduction de 1830 et ils sont considérés comme acquis dans celle de 1841. Pour en trouver une élucidation plus précise, il faut se reporter à la leçon X de I'Introduction historico-critique à la philosophie de la mythologie, probablement plus tardive, puisque la version dont on dispose a fait l'objet d'un enseignement donné à Berlin jusque dans les années 1845-1846, avec les révisions et les incessants remaniements dont Schelling était familier.

Qu'en est-il tout d'abord de la «philosophie de l'histoire » sur laquelle l'Introduction de 1830 passait très vite en se contentant de préciser qu'il allait de soi que la philosophie historique n'était pas une philosophie de l'histoire?

Dans l'Introduction historico-critique, après avoir rappelé que ce sont les Français puis Herder qui ont assuré la fortune de l'expression, Schelling avoue cependant une sorte

\footnotetext{
${ }^{3}$ COUSIN, V., SCHELLING, Victor Cousin: suivi de la correspondance Schelling - Cousin, « La véritable histoire de la philosophie attend l'accomplissement de la philosophie elle-même ", SW,VIII, 461 (idem in Lettre à Cousin du $1^{\text {er }}$ avril 1826), Centre d'Études d'Histoire de la Philosophie Moderne et Contemporaine, Paris, 1991.
} 
de perplexité. Parler de philosophie de l'histoire, c'est " définir l'Histoire comme un tout » (SCHELLING, Introduction à la philosophie de la mythologie 1998, 225). Or, objecte-t-il pour commencer, c'est un tout qui n'est pas tout puisque l'avenir n'en fait pas partie d'une part et que, d'autre part, ce tout ne sait pas lui-même où il commence. L'Histoire de la philosophie de l'histoire, c'est donc un tout qui ne commence ni ne finit nulle part, sans début ni avenir, « un tout (qui) progresse à l'infini, dépourvu de limites et donc de sens, un progrès sans arrêt et sans pause » (Ibidem, p. 226). Si lui fait défaut une véritable connexion des temps -et on devine que c'est dans cette direction que va se porter l'attention schellingienne-, cette histoire serait-elle du moins, dans ses contenus et ses objets, le "tout » du passé ? Ou bien, et c'est ce soupçon que porte Schelling, depuis le défaut constaté d'un système des temps, n'est-elle pas contrainte de considérer le passé lui-même sur le modèle de cette progression indéfinie, sans arrêt ni pause ? Qu'en est-il par exemple de la distinction usuelle entre le temps historique et le temps préhistorique ? Emporte-t-elle chez les historiens qui l'utilisent une différence d'essence, laquelle permettrait alors de produire des délimitations temporelles, des définitions effectives entre un temps proprement historique et un autre temps, un temps autre qu'historique ? Pourrait-on alors tracer une frontière réelle entre les deux ?

\section{Historicité et historialité}

Schelling montre que ce n'est nullement le cas. Cette distinction de l'historique et du préhistorique s'établit de façon toute triviale à partir d'une différence externe et contingente. Le temps historique dont parlent les historiens, c'est tout simplement celui dont nous pouvons savoir quelque chose. Le temps préhistorique, celui dont nous ne savons rien (Schelling s'amuse au passage de l'embarras où sont plongés les rédacteurs des grandes Histoires universelles encyclopédiques qui ne savent jamais où et par où commencer et ne peuvent donc jamais parvenir à un accord sur le commencement). Le temps préhistorique, il faudrait donc le nommer, suggère Schelling, préhistorial ou préhistoriographique (vorhistorisch) et non pas préhistorique (vorgeschichtlich) puisqu'il n'est rien de nonhistorique. C'est bien d'un même temps dont il s'agit en effet avec la préhistoire et l'histoire (des historiens), il n'y a strictement aucune différence interne, de contenu, entre les deux temps ainsi caractérisés. La fragilité des documents, des archives, la contingence radicale des modalités de leur attestation, leur crédibilité plus ou moins avérée le montrent bien. 
Mais est-ce que cette pseudo-différence, malgré tout, ne ferait pas signe tout autrement que selon la découpe entre une présence à elle-même de l'histoire d'une part et une antériorité à toute présence à soi dans le préhistorique d'autre part? II y aurait, nous dit Schelling, une différence plus ou moins sue, un préjugé plus ou moins avoué qui serait celuici : "les événements du temps préhistorique seraient insignifiants alors que ceux du temps historique seraient significatifs ". Or, c'est pratiquement l'inverse qu'il faut prendre très rigoureusement en considération. Ce qui « recèle les processus les plus significatifs ", c'est justement « cette contrée inconnue, cette région inaccessible à l'histoire, dans laquelle se perdent les sources ultimes de toute histoire (Geschichte) » (Ibidem, p. 227). Et si l'on veut à tout prix se mettre en quête d'une direction, d'un sens, d'un progrès, ils ne vont pas comme on croit, "du petit au grand", germinativement, c'est au contraire "le gigantesque qui constitue le commencement » biblique aussi bien qu'homérique.

C'est tout l'enjeu de la pensée schellingienne de l'histoire, de l'historique, de "l'histoire conçue comme telle en son historicité ${ }^{4}$, qui se montre ici. Cet enjeu est articulé philosophiquement autour de la question du commencement et de son statut (c'est-à-dire du rapport entre histoire et temps, entre historicité et système des temps), et il faut donc tâcher de penser une « effectivité absolue antérieure à toute possibilité, (une) effectivité qu'aucune possibilité ne précède ", un imprépensable (Ibidem, II, p. 111). Cet enjeu détient par conséquent dans son articulation un aspect également architectonique, autour de la place d'une philosophie de la mythologie comme «la partie la plus nécessaire et la plus incontournable " d'une pensée de l'Histoire, de l'historicité de l'histoire, à inventer ou à construire (SCHELLING, Introduction à la philosophie de la mythologie 1998, 232) depuis cet imprépensable ou cet immémorial. Sans doute faudrait-il ici exposer la philosophie de la mythologie proprement dite, et en particulier rappeler que le mythe, pour Schelling, n'est rien de mythique. Il est, justement, historique en tant qu'il a effectivement été. Mais je laisse cette exposition de côté pour aller directement à un certain nombre de traits.

Si la différence du temps historique et du temps préhistorique n'est guère intelligible selon la simple distinction externe inscrite dans un seul et même continuum temporel et qui n'affecterait pas véritablement ladite différence (et tel serait au fond le

\footnotetext{
${ }^{4}$ SCHELLING, Philosophie de la Révélation, trad. sous la dir. Courtine-Marquet, PUF, 1994, III,54 (SW,XIV,33 : es kommt darauf an, das Geschichtliche als Geschichtliche zu begreifen)
} 
préjugé foncier de toute philosophie de l'Histoire), de quelle nature peut-elle bien être en vérité ? On aurait affaire selon Schelling à deux temps essentiellement différents et discontinus que signalent de façon distordue les différences contingentes des historiens. Le contenu du temps préhistorique, c'est la krisis, le passage, la séparation différenciante, lesquels manifestent les mouvements de la conscience théogonique ("genèse des théologies ») et ethnogonique ("la séparation de l'humanité en peuples ») (Ibidem, p. 228). Ce temps préhistorique est donc rempli d'événements. II n'est pas vorgeschichtlich au sens où, en lui se passent (geschehen) toutes sortes de choses primordiales. II est tout aussi historique que le temps historique stricto sensu. Ce n'est pas là que se tient leur différence d'essence. Ce par où le temps historique se distingue essentiellement du temps préhistorique (mais on commence à voir que les superpositions ne coïncident plus, Schelling les fait considérablement bouger et les dénominations sont entièrement brouillées), c'est que désormais l'engendrement (le processus de séparation) prend figure d'engendré (de produit étant-là). La mythogonie appartient au passé en ce sens là. On n'a donc nullement à faire à un temps illimité et indistinct que I'historiographie pourrait à sa guise séquencer et segmenter selon le "degré de culture ", comme raille Schelling. "II y a des temps effectivement et intérieurement différents les uns des autres dans lesquels l'histoire se compose et $s^{\prime}$ articule $»^{5}$. Le temps propose, l'histoire dispose, en quelque sorte. Le temps dit historique reçoit ainsi une détermination, soit un «type » et une « loi » qui sont tout autres que le type et la loi du temps dit préhistorique. A son tour, ce temps préhistorique, déterminé lui-même par cette contre-détermination (qui oblige Schelling à l'appeler « relativement préhistorique »), s'adosse à quelque chose qui serait un temps non-temps selon l'expression de Rosenzweig, "absolument préhistorique ", "temps de la complète immobilité historique ", ne connaissant "aucune vraie succession de temps", "temps absolument identique ", "éternité relative", "sorte d'éternité " (Schelling renvoie ici à I'hébreu olam, temps-monde, Weltalter) (Ibidem, pp 229, 230). Une fois posé, donc, que le concept de Geschichte est plus "étendu " que celui d'Historie, le schéma proposé par Schelling distingue entre ces trois temps que seraient : 1 . le temps absolument préhistorique (temps d'avant toute histoire, aussi bien historiale qu'historique) ; 2 . le temps relativement préhistorique qu'il suggère d'appeler préhistorial (le temps des différentes -gonies), I'historique lato sensu; 3. et enfin le temps historique ou historial, l'historique stricto sensu.

\footnotetext{
${ }^{5} / d$. -souligné par moi.
} 
Des temporalités différentielles articulées en « histoire »

Quelles que puissent être les éventuelles objections qu'on opposerait à cette découpe, l'analyse schellingienne des structures de l'historicité paraît très féconde. Habermas sut l'apercevoir dans sa thèse non publiée, lorsqu'il suggéra que Schelling eut, de I'histoire, une "intelligence plus essentielle que Hegel » (HABERMAS 1954, 12). II semble même que l'auteur des Âges soit le co-inventeur du néologisme "Geschichtlichkeit " ou en tout cas, celui qui en assura les premières circulations, de même d'ailleurs que le Système de l'idéalisme transcendantal est à sa façon le premier texte où l'histoire est introduite comme thème et en tant que telle dans la philosophie transcendantale.

En tranchant comme elle le fait selon des qualités temporelles -tranchant qui lui confère ce cachet d'historicité singulier-, la pensée schellingienne de l'historique rompt avec les représentations d'un temps linéaire et homogène qui serait le temps indifférencié parcouru par l'Histoire, temps illimité pour une histoire temporellement indéterminée. Au contraire, par Schelling, le temps historique se trouve remarquablement inséré dans un " organisme, un système des temps ". Il est sans doute peu contestable qu'on puisse différencier plus et autrement que ne le fait l'Introduction historico-critique, mais ce qui importe, c'est la clé de l'organicité des temps et la direction qu'elle ouvre à la pensée de I'histoire dans et sous le temps. Chaque temps envisagé est délimité par un temps qui non seulement le précède ou le suit cumulativement, mais le contracte, le révoque et le métamorphose. Dans ce jeu de forces temporelles, les temps de l'histoire sont des temps proprement différents, historiquement distincts les uns des autres. II y a des temporalités historiques différentielles adossées elles-mêmes à ce "temps non-temps ", à l'immobilité d'une sorte d'éternité que les mytho-, ethno-, et théo-gonies zèbrent de leurs spécificités temporelles .

Une première remarque, ici, simplement allusive pour le moment : c'est à partir de ce schéma général que Rosenzweig envisagera pour son propre compte une véritable typologie des rapports entre ces temps (ou tel ou tel d'entre ces temps) et I 'éternité (ou la " sorte d'éternité » comme dit Schelling, c'est-à-dire, à chaque fois, une éternité imaginairement anticipée qui emporte une repensée du statut du commencement et du statut de la fin). Ce rapport du temps au temps non-temps, de la temporalisation à l'éternité, qualifie, du point de vue de Rosenzweig, une articulation singulière entre l'inscription 
particulière dans un temps historique donné et le mode singulier d'habitation de la temporalité. Le judaïsme et le christianisme en représentent les deux grands types étudiés dans l'Etoile de la rédemption, comme structures ou modalités de la relation du temps et de l'éternité, et plus profondément de l'insertion de l'éternité dans le temps.

A partir de la distinction de l'historicité et de l'historialité et, d'autre part, de la différence qualitative, essentielle, entre histoire proprement dite et préhistoire "critique » (au sens de la krisis qui préside à la mythogonie), Schelling fait un pas de plus et propose de lire l'histoire elle-même de façon stratifiée. L'histoire ordinaire (gemein), phénoménale, externe, serait de fait comme travaillée en son cœur par une histoire propre (eigentliche Geschichte) (SCHELLING, Les Âges du Monde 1992, 15), supérieure ou "suprême " (ein höheres Geschichtliches) (SCHELLING, Philosophie de la Révélation 2002, 41), interne, une " histoire supra-historique " selon une formule très remarquable ${ }^{6}$ qui, à bien des égards, fait écho, à quelque quarante années de distance à celle qu'on trouve dans le Système de l'idéalisme transcendantal d'un " progrès anti-historique ». Celui-ci, dans le texte de 1800, signifie la conjonction, plus ou moins kantienne, de deux éléments distincts: $a$. le concept d'histoire inclut le concept d'une progressivité infinie car il ne peut y avoir d'histoire (humaine) que sous la condition qu'un idéal fasse l'objet d'une transmission, moyennant "des écarts en nombre infini » dans la réalisation de cette transmission; $b$. cette progressivité, étant donné ce qu'elle est, sa nature, n'autorise nulle conclusion allant dans le sens d'une "perfectibilité infinie de l'espèce humaine " (SCHELLING, Système de I'Idéalisme Transcendental 1992, 225, 229). Ces formulations, "progrès anti-historique ", " histoire supra-historique ", ne sont nullement controuvées. Elles indiquent la permanence d'une tentative, tâtonnante mais insistante, de penser I'histoire tout autrement que sous le modèle des philosophies de l'histoire. En elles s'enracine un schellingianisme " de gauche » (qualification à prendre avec une immense précaution) dont deux noms m'apparaissent hautement exemplaires. Un penseur comme Moses Hess, qu'on range en bonne part à tort parmi les Jeunes-Hégéliens, proposait dans Die heilige Geschichte der Menschheit, un texte de 1837, de lire les événements historiques selon une double grille interne / externe structurée selon le pli différentiel du latent, les déterminations vécues du réel social, pour Hess, et du patent, leurs réalisations par défaut en quelque sorte. Ernst Bloch, le « Schelling

\footnotetext{
${ }^{6}$ Ibid., III, p. 56. Cf. également Les Âges du monde, éd. cit., p. 258 : « les monuments de l'histoire interne sont la principale source de l'histoire externe $"$.
} 
marxiste " d'Habermas, méditant quant à lui un passage du Zohar dans L'esprit de l'utopie, un texte à peu près contemporain de L'Etoile, fait de l'intervention alternée de deux temporalités différentielles décrites comme des espaces et des partages une clé fonctionnellement structurante requise par la compréhension de l'histoire. Je n'en dis pas plus, mais l'évocation de ces deux noms voudrait simplement suggérer qu'on a bien à faire à un ensemble ramifié, voire à un réseau de pensées, de dispositions et d'hypothèses dans lequel émerge une profonde considération de l'histoire en tant qu'elle demeure absolument intotalisable.

Si elle demeurait coupée de sa relation à l'histoire interne, l'histoire externe stagnerait dans une sorte de mouvement aveugle quant à la signification des processus qu'elle met au jour. Les événements qui la structurent, s'ils restaient déliés de tout rapport au supra-historique, sans relation vivante à une extra-historicité historique, pour le dire cette fois dans le registre rosenzweigien, ne seraient qu'égrénés à perte de vue, enchaînés causalement les uns aux autres, sans possibilité d'être déliés de cette concaténation, d'être rédimés si l'on peut dire, er-löst. Ils "tomberaient historiques", comme dit Péguy. Au fond, les sensibilités historiques les plus fines ( $c^{\prime}$ est ce qu'Habermas eut le mérite de repérer chez Schelling) s'attesteraient dans leur capacité à échapper à l'illusion funeste d'une pure immanence de l'histoire à elle-même. Penser quelque chose comme un au-delà de l'histoire, un supra- ou un extra-historique, c'est rappeler l'impossibilité stricte, autant ontologique que gnoséologique, d'une immanence absolue. L'histoire déterminée dans un rapport constitutif à une extériorité n'est donc pas Historie, masse accumulée d'événements qui se sont passés et qui se cristallisent en passé historique. L'histoire qui en établit la chronique forme une discipline légitime du savoir, Schelling le dit à plusieurs reprises, Rosenzweig en a été le praticien, mais elle n'épuise en aucune façon la considération proprement historique de I'histoire elle-même. L'historicité schellingienne -tel est le sens du terminus " historique » que l'idée d'une historicité supérieure permet de déterminer- désigne donc ce qui se donne aux hommes depuis une extériorité ou une altérité, quelque chose par conséquent qu'il leur faut proprement endurer. C'est bien la raison pour laquelle, comme l'aperçoit Schelling dès le Système de l'idéalisme transcendantal, I'histoire demeure réfractaire à la dimension de l'a priori et ne peut se constituer comme objet d'une 
philosophie à proprement parler, laquelle présupposerait une véritable doctrine de la détermination de l’agir humain dans sa prévisibilité

Le christianisme incarne, selon Schelling, et par l'Incarnation justement, l'entrelacs, le lien et le lieu où les deux histoires s'enchevêtrent. On pourrait aisément montrer que du côté du judaïsme (Hess ou Rosenzweig), la jonction-disjonction des deux histoires va s'opérer dans l'imbrication de deux mondes, de deux éons, "ce monde-ci » et le " mondequi-vient ", I'un dans l'autre, mais l'un radicalement distinct de l'autre, ces deux temporalités co-déterminant le monde lui-même comme un venir, ou un à-venir.

Pourquoi ce lien, profond, marqué, entre christianisme et historicité de la philosophie historique et positive schellingienne comme histoire supérieure -trans-, supraou extra-historique) ? D'abord parce que la philosophie positive prend en vue un avenir, un devenir, où se jouent des événements qui mettent en scène Dieu lui-même (création, chute, révélation, incarnation, rédemption). Le christianisme est en effet par soi-même ce qui vient rejouer l'archi-événement qui signifie la mise en mouvement, la propulsion historique inaugurale, à savoir l'expulsion du paradis. Cet Urereignis catastrophique est d'ailleurs toujours saisi comme un philosophème par Schelling. Le christianisme ébranle une deuxième fois le fond créationnel, il répète cet acte immémorial, lequel inaugure la série des événements qui en sortent. Et cette sortie, c'est l'histoire. Pour Rosenzweig, on a à faire là, sinon à une fausse sortie, tout au moins à une échappée qui demeure consubstantiellement liée à cela à quoi elle échappe. Aussi sa fonction, si éminente soit-elle puisqu'elle fournirait selon Schelling la signification même du christianisme, n'empêche nullement que " toute histoire n'est que préhistoire ${ }^{7}$ et que nous soyons encore dans l'avant toute histoire, dans l'avant toute venue. Cette différence, où se condense celle du christianisme et du judaïsme, ne peut masquer l'importante concordance autour de l'idée d'une "deuxième fois " profondément méditée par Rosenzweig, qui en fait la marque, l'insigne le plus distinctif de toute réalité effective (ROSENZWEIG, Foi et Savoir 2001, 187). La deuxième fois renouvelle la première et sans ce renouvellement il n'y aurait même pas de première fois, mais simplement un être qui ne pourrait jamais s'arracher à soi et se hausser ainsi à l'existence. Einmal ist keinmal, dit l'adage allemand. Une fois, c'est comme jamais, et au sens fort, ça

\footnotetext{
${ }^{7}$ ROSENZWEIG, Etoile et Rédemption, p. 308. La formule est étonnamment proche de l'un des leitmotive de l'anthropologie philosophique du jeune Marx.
} 
n'existe pas. L'existence juive, pour Rosenzweig, est tout entière dans l'attente de la deuxième fois du renouvellement, tout comme le christianisme, pour Schelling, est au principe de la série historique des effets de la deuxième fois de l'Incarnation.

C'est d'ailleurs à ce titre qu'il peut être dit, à bon droit, " religion éminemment historique » et qu'il emporte quelque chose de capital, à savoir une « identité du doctrinal et de l'historique ": " ce serait une mauvaise explication du christianisme, une suppression complète de ce qui lui est propre, que de vouloir distinguer le doctrinal et l'historique et considérer seulement le premier comme l'essentiel, le contenu propre, mais l'historicité comme une simple forme ou un revêtement. L'historicité n'est pas quelque chose de contingent pour la doctrine, elle est la doctrine même " (SCHELLING, Philosophie de la Révélation 2002, 41). Par là, se marque très nettement la différence foncière entre philosophies positive et négative.

\section{Qu'est-ce qu'une philosophie historique?}

Quelle est en effet aux yeux de Schelling le trait le plus remarquable de la philosophie négative ou purement rationnelle? C'est de bannir tout élément historique de son explication du monde : elle est anhistorique, intemporelle et éternelle, dit la note de 1841 en se rapportant au mos geometricus spinozien. Si la philosophie positive peut en revanche être dite historique, c'est parce qu'elle s'oriente principalement sur le fait, l'acte, l'événement, le quod ou le $d a \beta$ en tant qu'ils ne se laissent pas appréhender a priori dans l'élément d'un penser. La philosophie de la mythologie constitue le fond où l'« identité du doctrinal et de l'historique » est déjà mise en évidence. La philosophie de la révélation prend en charge cette identité en la mobilisant en quelque sorte, au sens où elle est irruption d'un " monde nouveau de mobilité » (Ibidem, p. 242), irruption d'un mouvement sur fond de cet imprépensable sans lequel il n'y aurait pas même d'histoire. Ce Grund, ce commencement dont Schelling fut "l'obsédé " (X. Tilliette), fonde, en même temps que la possibilité d'une histoire, la succession elle-même, le principe temporel qui permet cette possibilité. Le fond assure en effet un passé puisqu'il vient avant le fondé qu'il fonde. Par lui, « un sujet peut être posé à la fois comme étant ceci et ayant été cela, c'est-à-dire comme possédant une histoire » (MARQUET 1973, 395). Et ceci vaut de la même façon pour l'humanité en son entier, dans son histoire qui va de la mythologie à la révélation comme blocs de positivité et d'historicité. La distinction/relation entre histoire "ordinaire » et histoire "supra- 
historique " permet une attention particulière aux phénomènes de l'histoire empirique, une vraie sensibilité historique, capable à la fois de penser l'action consciente et projective des hommes et l'opacité profonde, insondable, de ses effets. On pourrait dire, comme le fait Schelling pour l'art dans le Système de l'idéalisme transcendantal, qu'il y a pour les sujets historiques un moi qui serait conscient selon la production et inconscient du point de vue du produit. L'Histoire des philosophies de l'histoire, en revanche, est comme ce « deuxième monde " dont parle Schelling dans les premières pages, très mélancoliques, de l'Introduction de Berlin de 1841, surélevé au-dessus de la nature par les hommes qui en sont les initiateurs et les fondateurs. Deuxième monde dont on pourrait attendre, puisque les hommes y habitent et s'y meuvent par la liberté de leur volonté, qu'ils y acquièrent le sentiment de "commencer pour eux-mêmes une série nouvelle d'événements ». Or ils traversent ce deuxième monde dans l'opacité et l'étrangeté à soi car ce monde est pour eux comme " un effrayant mouvement perpétuel (que nous nommons I'histoire) » (SCHELLING, Philosophie de la Révélation 2002, 24).

Je voudrais avancer quelques conclusions brèves, d'abord en ramassant ce que j'ai dit de Schelling, et en ouvrant ensuite deux ou trois questions dans la perspective qui est celle de Rosenzweig.

1. L'histoire, chez Schelling, est soumise à son articulation selon des temporalités spécifiques et distinctes et son temps lui-même est adossé à un temps intemporel qui forme le fond de toute temporalité et de toute histoire. Cette plasticité ouvre à la possibilité de penser l'éternité elle-même comme une catégorie historique, ou en tout cas comme une catégorie sur laquelle l'historicité peut venir se brancher, ce qu'on retrouve précisément chez Rosenzweig.

2. Si nulle philosophie de l'histoire n'est autorisée par ce préalable (il me semble d'ailleurs que la philosophie positive interdit toute " philosophie de »), une véritable pensée de l'histoire peut être déterminée chez le dernier Schelling. Elle n'entendrait nullement en saisir la prévisibilité, la Gesetzmässigkeit, mais accueillir l'histoire ou l'historicité comme ce par quoi se fait connaître à l'expérience un imprévisible, un imprépensable, la factualité d'un fait, d'un factum, de l'existence, qui excède la raison historique.

3. On comprend évidemment que cette histoire " expériencielle " ne se laisse pas représenter dans une téléologie de la liberté qui se résorberait librement dans une 
nécessité dès lors que l'avenir est envisagé comme autoréalisation et autoreprésentation de la liberté dans le mouvement de l'Idée. Loin de l'épopée d'un sens, loin de se disposer selon une structure sensée et selon l'unification possible des fins qui l'accompagne, l'histoire, ici, ne se déroule pas vraiment selon une évolution; elle se surprend sans cesse en effectuant l'événement de ce qui ne cesse d'advenir dans sa Wirklichkeit.

4. Le mérite de la position schellingienne, c'est peut-être d'échapper à l'opposition ou à l'alternative convenue entre, d'un côté, une histoire consciente de soi, une histoire de l'autolibération par la réflexion et, d'un autre côté, une histoire qui ne serait que le pur déchaînement de forces totalement aveugles, qui ne serait qu'interminable bruit et fureur. L'acte fondatif et inaugural de l'histoire, dans le procès théogonique où s'inscrivent chute, sortie, exil, est donc un mouvement que les hommes ne peuvent reconnaître comme quelque chose qu'ils auraient accompli. L'histoire avance, mais sans voir. Elle s'avance sans se voir selon un "progrès anti-historique » qui l'apparente partiellement à l'ange benjaminien des thèses sur le concept d'histoire.

Les quatre conclusions précédentes invitent à revenir sur l'opposition symétrique déjà indiquée tout à l'heure et qui n'est, à vrai dire, que purement formelle, entre le caractère " éminemment historique » du christianisme selon Schelling et l'extra-historicité métaphysique du judaïsme selon Rosenzweig. Cette extra-historicité du "peuple éternel » consiste en une extériorité radicale, en une séparation d'avec la temporalité du monde, celle de l'histoire des peuples et des peuples dans l'histoire. La séparation en extériorité qui consiste pour Rosenzweig en un retournement très subtil des analyses "théologiques " du jeune Hegel dans sa période francfortoise se marque, selon les descriptions de l'Etoile, dans la non-autochtonie, la migration, le passage et la quasi-installation du " peuple juif » dans l'éternité anticipée par la symbolique de la Loi et de son intemporalité. Ainsi, le jeu du judaïsme avec le monde ou avec l'histoire et ses avatars, pour dire la chose ainsi, traverse la distinction d'un dedans et d'un dehors de l'histoire en la réfractant au sein de soi, dans la différence interminable entre les " destinées extérieures », soit le judaïsme engagé dans les aventures du siècle, le sionisme, l'assimilation, et le "reste " de sa croissance par soustractions.

On peut avancer que, dans les analyses rosenzweigiennes, l'extériorité du judaïsme ne s'affirme comme telle que par rapport à la Weltgeschichte hégélienne. Si l'on retient 
I'hypothèse théorique schellingienne d'une histoire supra-historique, elle prend une tout autre coloration. Au fond, on le comprend, et Schelling et Rosenzweig construisent de façon très consistante des modèles théoriques concurrents du modèle hégélien. Ce qui, pour tous deux, fait problème dans l'ontologie hégélienne de l'histoire, c'est en profondeur le rapport entre histoire et temps. Pour fonder l'intelligibilité de l'histoire, Hegel part de l'éternité de I'Idée, de l'intemporalité du processus logique. Ce qui oblige à admettre que pour concevoir I'histoire, au sens fort, il faille abolir le temps, ou, à tout le moins, se soustraire en pensée au temps. Le triomphe du concept emporte en quelque sorte la cessation du temps : le premier est le second "étant-là ", selon la fameuse formule de la Phénoménologie de l'esprit. Le temps, c'est le concept en tant qu'il est là, en tant qu'il existe. II s'en suit ou il peut en tout cas s'en suivre une certaine confusion entre l'histoire effective, la suite des événements effectifs, et les structures de l'historicité, les conditions transcendantales de son effectuation. Schelling met explicitement en garde contre cet écrasement d'un Wirkliches sur la Wirklichkeit elle-même, à savoir sur le mode de surgissement d'un effectif (SCHELLING, Philosophie de la Révélation 2002, 83). S'il doit être bien clair que l'hégélianisme n'est en aucune façon un dogmatisme méta-historique, ni une théologie de I'histoire qui figerait l'effectivité, la Wirklichkeit, sous son idéalité dialectique et rationnelle, le problème de l'intemporalité des structures de l'historicité qui en assure l'intelligibilité comme automouvement demeure entier. Sous ce considérant, qui n'est pas mince, la philosophie hégélienne de l'histoire se présente comme une philosophie du devenir -mais d'un devenir qui n'est pas lui-même devenant puisqu'il est à son tour frappé d'intemporalité. On pourrait ainsi distribuer les dispositions ontologico-historiques: d'un côté une philosophie de l'histoire comme philosophie du devenir sans "préhistoire " et téléologie subjective de la liberté; d'un autre côté une philosophie du temps et de l'événement, soit de ce qui ne peut en aucune façon être prépensé, de ce dont la réalité prévient la possibilité, jusqu'à l'excès éventuel du messianisme.

La trop fameuse question de la fin de l'histoire, par-delà les contresens et les lourds malentendus qu'elle ne cesse de transporter, demeure ici, pour notre propos, très symptômale. II va de soi qu'elle ne saurait signifier l'arrêt de l'histoire, ni la visée consciente d'un but final -c'est en ce sens que je disais de la téléologie hégélienne qu'elle n'est pas une téléologie objective, mais subjective. En quoi le thème de la fin de l'histoire fait-il alors 
symptôme ? En cela qu'il indique très fortement que l'histoire, si on ne veut pas se contenter de la représenter comme une accumulation anecdotique de choses sans intérêt, doit avoir une structure sensée. Bien sûr ce sens ne se dispose pas chez Hegel une fois pour toutes et selon une universalité abstraite et ineffective. Mais, toutefois, il retotalise le passé, ou $d u$ passé, dans le présent, avec le risque, jamais vraiment conjuré, que l'avenir soit envisagé comme l'autoréalisation de cette structure sensée dans une possible unification des fins. Et comme le système est un système de la liberté, on peut dire que le savoir absolu implique un achèvement de l'histoire au moins en tant que recherche de la liberté de l'esprit.

L'extra- ou le supra- (l'extériorité et la supériorité) sont donc pensés par Rosenzweig et Schelling comme des propositions contre-hégéliennes mais affirmatives destinées à servir à une critique résolue du temps historique homogène et autodéterminé, linéaire et sans dehors, telle que l'emporte l'ontologie hégélienne de l'histoire. II est important de rappeler l'immense mérite de Schelling dans la formation, c'est-à-dire dans la mise en forme et l'articulation théorique de cette critique.

Autant que l'extériorité rosenzweigienne, le caractère " éminemment historique » du christianisme désigne une extériorité par rapport à l'histoire " ordinaire » (c'est-à-dire sans extériorité, justement), une transcendance. Là se tiendrait son éminence, l'animation d'une histoire interne qui battrait au coeur de l'histoire ordinaire, externe. De même, pourrait-on dire, l'extra-historicité juive n'est-elle pas sans effets historiques, puisque sa dimension utopique, qui ne va pas sans "périls", Rosenzweig le souligne maintes fois, emporte une critique de toute substantialité historique, de l'Etat en particulier. Penser une dimension non-historique inscrite ou désinscrite dans la trame de l'histoire, une extériorité à..., c'est évidemment poser d'emblée la question du rapport à ce dont l'extériorité s'extériorise, d'un rapport à l'histoire par conséquent, mais d'un tout autre type que celui de la Weltgeschichte, hétérogène à tous les modèles, de provenance plus ou moins hégélienne, de la réalisation et de l'autoréalisation.

On peut se demander en outre si le caractère éminemment historique du christianisme (au sens, on l'a compris désormais, d'une historicité supérieure, intérieure, subtile) relevé par Schelling ne répond pas au fond à ce qu'en repère Rosenzweig lui-même dans toutes une séries d'analyses et de métaphores qui servent, dans l'Etoile, à mettre en concurrence les deux modèles juif et chrétien de la rédemption : l'arche sur le fleuve et le 
rail qui le longe, les rayons qui se propagent et le cœur ardent. C'est en bonne part le cas. Mais il ne s'agit pas pour Schelling, avec le christianisme, d'une religion dans l'histoire au sens où Rosenzweig en détermine la dialectique historique et temporalisante. II y va bien plutôt d'une religion qui libère l'historicité, qui la rend possible en quelque sorte, depuis cette idée d'une mobilisation, d'une mise en mouvement, d'un archi-événement qui serait le commencement de toute événementialité. Ceci, si on devait le prendre en compte radicalement et jusqu'au bout, complexifierait le modèle étoilé de Rosenzweig, le cœur et les rayons.

C'est sans doute la révélation comme structure fondamentale de l'extériorisation, de toute extériorisation, qui rassemble ces deux pensées. L'être schellingien, comme la création rosenzweigienne, constituent des modes de non-existence, de pure "première fois " privée de tout renouvellement. Exister, en ce sens, c'est tout bonnement se révéler et on voit bien comment les catégories traditionnelles de la théologie peuvent être convoquées par les deux pensées de l'existence. Ce qui se contenterait d'être ou même d'être-là n'existerait pas. Un Absolu non historique tout comme une création sans révélation dessineraient deux figures de ce que Rosenzweig nomme génériquement paganisme. II faut un arrachement de soi à soi, un surmontement d'un fond pour qu'un Existant se mette à exister sur fond de cette déposition de soi, d'exposition hors de soi. En ce sens, affirmer que Dieu existe, ce ne peut être autre chose que de dire : il s'est révélé, il s'est extériorisé pour un autre que soi. Autant l'acquis schellingien de la critique kantienne de l'argument ontologique, en tant qu'il constitue une structure de l'existence, que le modèle de la Kabbale lourianique d'un auto-anéantissement de Dieu repris par Rosenzweig dans le thème d'une auto structuration interne de Dieu préalable à toute création, autant l'un que l'autre, donc, peuvent ici servir à mieux comprendre qu'à toute histoire aussi il faut un devenir-manifeste, une révélation, une production d'extériorité, pour qu'elle soit seulement intelligible comme histoire. Pas d'historicité pensable, donc, sans que le modèle structural de la révélation, de l'ouverture à l'extériorité, n'entre en jeu de façon décisive. C'est sans doute là que les deux penseurs se retrouvent dans une affinité stellaire qui n'exclut jamais la profondeur des différences.

\section{REFERENCES}


COUSIN, victor, SCHELLING. Victor Cousin: suivi de la correspondance Schelling - Cousin. Paris: Centre d'Histoire Moderne et Contemporaine, 1991.

HABERMAS, Jürgen. "Das Absolute und die Geschichte im Denken Schellings." Research Gate. 1954. http://www.researchgate.net/publication/35189039_Das_Absolute_und_die_Geschichte_im_Denke n_Schellings (accessed 2014).

MARQUET, Jean-François. Liberté et Existence - Étude sur la formation de la Philosophie de Schelling. Paris: Gallimard, 1973.

ROSENZWEIG, Franz. Étoile et Rédemption. Paris: Seuil, 2003.

-. Foi et Savoir. Paris: J.Vrin, 2001.

SCHELLING. Contribution à l'histoire de la philosophie moderne. Paris: PUF, 1983.

-. Introduction à la philosophie. Paris: Jean Vrin, 1996.

-. Introduction à la philosophie de la mythologie. Paris: Gallimard, 1998.

—. Les Âges du Monde. Paris: Ousia, 1992.

-. Philosophie de la Révélation. Paris: PUF, 2002.

—. Système de I'Idéalisme Transcendental. Paris: Ousia, 1992. 